\title{
Le Siècle du sexe ? Genre, corps et sexualité au dix-huitième siècle (vers 1650-vers 1850)*
}

The century of sex? Gender, bodies, and sexuality in the long eighteenth century

\section{Karen Harvey}

\section{(2) OpenEdition}

\section{Journals}

Édition électronique

URL : http://journals.openedition.org/clio/9683

DOI : $10.4000 /$ clio.9683

ISSN : 1777-5299

Éditeur

Belin

Édition imprimée

Date de publication : 1 mai 2010

Pagination : 207-238

ISSN : 1252-7017

Référence électronique

Karen Harvey, «Le Siècle du sexe ? Genre, corps et sexualité au dix-huitième siècle (vers 1650-vers

1850)* „, Clio. Femmes, Genre, Histoire [En ligne], 31 | 2010, mis en ligne le 31 mai 2010, consulté le 20 avril 2019. URL : http://journals.openedition.org/clio/9683; DOI : 10.4000/clio.9683

Tous droits réservés 


\section{États de la recherche}

\section{Le Siècle du sexe ? Genre, corps et sexualité au dix- huitième siècle (vers 1650-vers 1850)*}

Karen HARVEY

L'article qui suit est paru en 2002 sous le titre A century of Sex : gender, bodies and sexuality in the long eighteenth-century dans The Historical Journal (vol. 45, 4, p. 899-916). Il fait le point sur la réception de la thèse de Thomas Laqueur par les historiens britanniques. Il présente également les principaux ouvrages consacrés au corps et à la sexualité au XVIII siècle depuis les xuvres pionnières de Lawrence Stone et Edward Shorter jusqu'à celles parues au début des années 2000, attirant ainsi l'attention sur la continuité et la vitalité de l'bistoriographie anglaise sur la question.

L'engouement pour le dix-huitième siècle - un "long" dixhuitième siècle compris dans des bornes chronologiques larges - peut en partie être mis sur le compte d'«un mythe britannique étrangement persistant»: cette période «aurait offert un terrain de jeu sexuel dénué de culpabilité, peuplé de belles jeunes filles et de coureurs fanfarons prêts à se rouler dans le foin dès qu'un mouchoir tombait au sol $»^{1}$. Pour les historiens professionnels également, il

* Je remercie Sandra Cavallo, Mark Jenner, feu Roy Porter, Lyndal Roper, Nick Stargardt, et particulièrement Amanda Vickery et Penelope J. Cornfield pour leur 
s'agit $d u$ siècle du sexe et du corps. Roy Porter a intégré la sexualité dans sa désormais classique histoire sociale de la période, indiquant que «parmi les riches et les oisifs, la sexualité se dégelait. La libido se libérait $»^{2}$. L'historiographie anglaise décrit le dix-huitième siècle comme l'un des temps forts de la libération sexuelle. Roy Porter a aussi avancé l'idée, dans des travaux ultérieurs, qu'à l'époque des Lumières la mise en valeur du corporel a généré une prodigieuse «libération hédonistique de la libido», reprenant ainsi une formulation antérieure de Lawrence Stone selon laquelle «la liberté d'expression sexuelle a été l'un des nombreux effets induits de la quête du bonheur au dix-huitième siècle $»^{3}$. Certes, on a tendance à penser que les genres littéraires intuitivement perçus comme les expressions les plus significatives des attitudes sexuelles d'une culture donnée, à savoir la littérature érotique et la littérature pornographique, font partie intégrante de l'époque des Lumières en Angleterre et en Europe ${ }^{4}$. Robert Darnton a soutenu la thèse que la littérature clandestine - y compris pornographique - avait largement contribué à la Révolution française ${ }^{5}$. Une grande partie des travaux sur les idées nouvelles des Lumières repose sur la place centrale qu'occupe la catégorie de genre. La révolution scientifique, les disciplines ethnographiques et médicales, par exemple, ont été en partie construites sur (et renforcées par) les notions de différence sexuelle et de rôles sexués; les travaux qui démontrent le caractère sexué des nouvelles formes de connaissances et de pratiques ont intégré les études sur le corps et sur sa signification culturelle ${ }^{6}$. Le corps est non seulement incorporé à l'historiographie des Lumières mais, dans certains cas, il est présenté comme typique de la modernité du dix-huitième siècle.

lecture des premières ébauches. Ce travail a reçu le soutien de la William Andrews Clark Memorial Library et de la British Academy.

1 Tonkin $1996: 2$.

2 Porter 1982a : 278.

3 Porter 1982b : 5. Voir Stone 1979 : 328.

$4 \quad$ Wagner 1988 ; Hunt 1993.

5 Darnton 1995a et 1995b. Voir également Chartier 1993.

6 Voir, par exemple, Jordanova 1989 ; Merchant 1980. 
En effet, l'historiographie de la sexualité et du corps est aujourd'hui un terrain où sont débattues des questions anciennes propres à la période - en particulier, celles qui concernent la modernité et l'Ancien régime 7 . Les histoires du corps et de la sexualité se concentrent sur la période qui court de 1650 environ à 1850 et insistent tout particulièrement sur le dix-huitième siècle; par ailleurs, elles décrivent cette période comme le siècle du changement : la conception du corps, la construction de la sexualité, l'activité sexuelle ont alors été soumises à des bouleversements. Décrit comme «l'ouvrage d'histoire médicale qui a probablement eu le plus d'influence au cours des vingt dernières années », La Fabrique du sexe (1992; 1990 pour la version originale en anglais) de Thomas Laqueur intègre l'histoire du corps et de la sexualité à une vision modernisante du dix-huitième siècle. Laqueur soutient qu'«à la fin ou vers la fin du dix-huitième siècle $»^{8}$, s'est produit un changement dans la façon dont le corps humain a été conçu. Avant le dix-huitième siècle, les hommes et les femmes étaient placés sur un axe vertical, hiérarchique, au sein duquel leurs corps étaient perçus comme deux variantes comparables d'un seul et même type. Le système humoral était le socle de ce «modèle unisexe » et, dans ce système, le corps était représenté comme une combinaison de quatre humeurs de qualités différentes froide, chaude, humide et sèche. Selon cette conception physiologique du corps, les individus présentaient des variations dans leur composition humorale. En conséquence, bien que les femmes fussent toujours dominées par des humeurs froides et humides, et les hommes par des humeurs chaudes et sèches, les différences de sexe étaient appréhendées comme des différences de degré. C'est dans les homologies structurelles des parties génitales masculines et féminines qu'on en trouve l'exemple le plus frappant: «le vagin est imaginé comme un pénis intérieur, les lèvres sont l'équivalent du prépuce,

7 Pour l'Ancien régime voir Clark J.C.D. 1985 et 2000. Pour des études historiques qui mettent l'accent sur la "modernité", voir, par exemple, McKendrick, Brewer \& Plumb 1982 ; Ogborn 1998.

8 L'évaluation du livre de Laqueur est tirée de Jenner \& Taithe $2000: 191$. Laqueur 1992 : 18 (en anglais Laqueur $1990: 5$ ). 
l'utérus du scrotum et les ovaires des testicules »9. C'est après le dixhuitième siècle que, d'après Laqueur, le "modèle des deux sexes" s'imposa. Les femmes et les hommes furent alors disposés horizontalement : on souligna leurs différences d'anatomie et l'on se mit à considérer leurs corps comme qualitativement distincts. De plus, un changement se produisit non seulement dans la proportion relative des différences ou des similitudes, mais aussi dans la conception de la nature qualitative des corps. Ainsi, dans le monde du sexe unique, "il faut à mon sens comprendre le sexe, ou le corps, comme l'épiphénomène, tandis que le genre, ce que nous prendrions pour une catégorie culturelle, était premier ou "réel" [...]. Autrement dit, avant le dix-septième siècle, le sexe était encore une catégorie sociologique et non ontologique ${ }^{10}$, écrit Thomas Laqueur. C'est au dix-huitième siècle que le sexe moderne est né et, au dix-neuvième siècle, la différence des sexes est désormais perçue comme le produit des nerfs, de la chair et des os.

Thomas Laqueur a pris soin de préciser que le changement ne s'est pas fait sans à-coup ni de manière définitive, et il a rassemblé des preuves qui attestent de «la permanence du modèle unisexe $»^{11}$ aux dix-neuvième et vingtième siècles. Mais peu d'historiens de la période moderne s'intéressent aux derniers chapitres de La Fabrique du sexe, qui soulignent la vigueur de la pensée unisexe dans les débats sur la masturbation et la prostitution ainsi que dans les thèses de Freud sur la sexualité clitoridienne et vaginale. Si l'ouvrage en vient peut-être à faire une place trop grande au changement intervenu au dix-huitième siècle, les derniers chapitres de La Fabrique du sexe occupent non seulement une part relativement peu importante du livre, mais ils présentent des études de cas qui contrastent avec la vue d'ensemble plus riche et plus ample qui est tracée de la pensée qui était dominante durant la période moderne ${ }^{12}$. Un certain nombre de formulations stipulant qu'un modèle unisexe a perduré « jusqu'aux

9 Laqueur 1992 : 17. Voir pour plus de détails p. 41-54 (en anglais Laqueur 1990 : 4 et 23-35).

10 Ibid. : 21-22 (en anglais : 8 ).

11 Ibid. : 37 (en anglais : 21).

12 Ibid. : 260-282 (en anglais : 227-243). 
alentours de 1700 », que «le modèle un seul sexe/une seule chair a dominé la réflexion de la différence sexuelle de l'Antiquité classique jusqu'à la fin du dix-septième siècle » et qu'«à la fin du dix-septième siècle et au dix-huitième siècle, la science donna une substance [...] aux catégories de "mâle" et "femelle" envisagés comme des sexes biologiques opposés et incommensurables", présentent sans aucun doute la période comprise entre 1700 et 1800 comme une étape clé dans cette évolution chronologique ${ }^{13}$. Par conséquent, ce qui ressort de La Fabrique du sexe, c'est une thèse vigoureuse sur le changement. Alors que les conclusions de Thomas Laqueur reposent en grande partie sur la lecture d'ouvrages médicaux et philosophiques et, en particulier, de ceux qui étudient l'anatomie et la physiologie reproductrice, l'auteur réfute avec force le fait que des progrès scientifiques aient pu être à l'origine du glissement du modèle unisexe vers le modèle des deux sexes. Il faut attendre le dix-neuvième siècle pour qu'interviennent plusieurs découvertes fondatrices. Comme Thomas Laqueur l'explique, «après la révolution scientifique, la différence sexuelle ne procéda pas plus de l'anatomie que ça n’avait été le cas dans le monde du sexe unique » ${ }^{14}$. En fait, "différence et identité, plus ou moins mystérieuses, sont partout; mais c'est hors des limites de l'investigation empirique que se déterminent lesquelles comptent et à quelles fins $»^{15}$. Discutant la notion de changement, Laqueur explique que :

[...] en eux-mêmes, les changements politiques et sociaux n'expliquent pas la réinterprétation des corps. L'essor de la religion évangélique, la théorie politique des Lumières, le développement de nouveaux espaces publics au dix-huitième siècle, les idées lockéennes sur le mariage envisagé comme un contrat, les possibilités cataclysmiques de changement social qu'entraîna la Révolution française, le conservatisme postrévolutionnaire, le féminisme postrévolutionnaire, le système des fabriques avec sa restructuration de la division sexuelle du travail, l'essor d'une économie de marché de services et de marchandises, la naissance de classes, isolément ou de manière solidaire, rien de tout cela ne fut la cause de la formation d'un nouveau corps sexué. Le fait est plutôt que la

13 Ibid. : 18, 42, 176 (en anglais : 5, 25, 154).

14 Ibid. : 186 (en anglais : 163).

15 Ibid. : 24 (en anglais : 10). 
reformation du corps se trouve intrinsèquement inscrite en chacune de ces évolutions ${ }^{16}$.

Laqueur propose donc une explication à multiples facettes. Pourtant, dans La Fabrique du sexe, il apparait que l'élément déclencheur du changement est un impératif politique-adopté par les scientifiques - destiné à réévaluer les corps afin de stabiliser et de préserver un ordre social fondé sur l'inégalité de genre. Alors qu'au dix-huitième siècle, les penseurs politiques ont de plus en plus eu recours à un discours porteur d'une égalité potentielle, fondé sur le droit naturel, la définition de la "femme" se devait de la poser comme qualitativement différente des hommes afin de tenir le pouvoir politique hors de sa portée ${ }^{17}$. La science a arbitré un débat politique sur les droits de l'homme, démontrant qu'il y avait des différences fondamentales au sein du genre humain qui justifiaient l'inéquité entre hommes et femmes dans l'accès au pouvoir. Comme Londa Schiebinger l'a également indiqué, «les droits naturels de l'homme n’ont pu être contrecarrés que par la preuve qu'il y avait des inégalités naturelles $»^{18}$.

Le travail de Laqueur peut être situé dans un large éventail de contextes historiographiques, mais il a eu beaucoup d'influence sur l'histoire du genre. Si le genre est défini comme « le savoir qui attribue des significations aux différences corporelles $»^{19}$ (selon la formulation de Joan Scott), alors la cartographie de l'histoire des conceptions de la différence sexuelle dressée par Thomas Laqueur est perçue à juste titre comme une contribution majeure à l'histoire des hommes, des femmes et du genre. En effet, les historiens des femmes et du genre ont intégré le récit laqueurien à un tableau plus vaste des relations de genre et de l'expérience sexuée. Cela tient en partie au fait que Thomas Laqueur a élaboré son argumentation à partir d'hypothèses tirées de deux domaines: la sexualité féminine et la relation des femmes avec la sphère "privée". Pour Laqueur, "la première étape incontournable» dans le passage du modèle unisexe au modèle des

\footnotetext{
16 Ibid. : 25-26 (en anglais : 11).

17 Ibid. : 221-238 (194-207).

18 Schiebinger $1993: 143$. Voir aussi ead. $1989: 216$.

19 Scott $1988: 2$ («Introduction »).
} 
deux sexes au dix-huitième siècle est un changement dans les conceptions de la procréation ${ }^{20}$. Une des conséquences de ce changement est la «relégation de l'orgasme féminin» dans les discours médicaux du dix-huitième siècle ${ }^{21}$. Dans la théorie aristotélicienne traditionnelle de la génération, où il n'y a qu'une seule semence, les femmes ne produisent pas de semence si bien que l'éjaculation féminine n'est pas nécessaire ; la conception a lieu quand la substance active de l'homme agit sur la substance passive de la femme contenue dans son sang menstruel ${ }^{22}$. Ceci a été remis en cause plus tard par la domination de la théorie hippocratique et la théorie galénique des deux semences. Même si cette dernière attribue à la semence de la femme un rôle moins important, dans ces deux théories les deux semences sont nécessaires pour agir sur la matière secrétée par la femme ${ }^{23}$. Dans le modèle unisexe, les théories où il y a dualité de semence dominent: «les deux sexes éprouvaient au cours des rapports un violent plaisir en étroite relation avec une heureuse génération ; tous deux émettaient généralement quelque chose $»^{24}$. Au cours du dix-huitième siècle, cependant, les théories où deux semences étaient nécessaires à la reproduction ont été remises en cause et l'on a fini par considérer que le plaisir sexuel de la femme symbolisé par l'orgasme féminin-n'était pas essentiel à la conception. Ce qui participait d'un changement de perception de la nature passive de la sexualité des femmes, changement analysé par Nancy Cott dès 197825. Comme l'explique Ruth Perry, «Historiquement, les femmes ont été perçues comme des créatures lascives et avides [...]; au milieu du dix-huitième siècle, on les décrivit de plus en plus comme appartenant à un autre ordre d'existence : capables d'aimer mais sans besoins sexuels $»^{26}$. Carolyn Merchant a établi un lien entre les changements intervenus dans la

\footnotetext{
20 Laqueur $1992: 22$ (en anglais : 8).

21 Laqueur $1986: 3$.

22 McLaren 1985 : 326 ; Bullough 1973 : 485-501.

23 Ainsi, on disait parfois que les femmes étaient capables de s'auto-féconder sans avoir recours à l'homme. Voir Crawford 1994 : 90.

24 Laqueur 1992: 67 (en anglais : 46).

25 Cott $1978: 219-236$.

26 Perry $1992: 212$.
} 
perception de la nature durant la révolution scientifique et la réaffirmation du rôle passif des femmes dans la reproduction, le refoulement simultané de la "passion sexuelle" et l'association croissante des femmes des classes moyennes et supérieures avec la vie domestique $^{27}$. De la même manière, Angus McLaren a montré qu'aux descriptions présentes dans la littérature médicale des seizième et dixseptième siècles, où la sexualité féminine était perçue comme valide, nécessaire et bien "terrestre », ont succédé celles de "victoriennes passives, allongées sur le dos et ne pensant qu'à l'Empire ». Comme Carolyn Merchant, Angus McLaren établit un lien entre ce récit de l'évolution de la sexualité féminine et la création «d'une nouvelle image de la femme de la classe moyenne, respectable et asexuée $»^{28}$. Depuis plusieurs décennies, les historiens des femmes rendent compte de l'émergence d'un nouvel idéal de féminité qui a confiné les femmes, devenues chastes, modestes et maternelles, au sein d'une famille de plus en plus privatisée ${ }^{29}$. Reprenant des travaux anciens sur l'expansion industrielle, les historiens des femmes ont avancé l'idée que l'expérience féminine s'est radicalement transformée quand les femmes ont été chassées du monde du travail et qu'elles ont été cantonnées à la sphère domestique de la maison ${ }^{30}$. Ainsi, les nouvelles interprétations de la sexualité féminine sont-elles intimement liées aux nouveaux rôles assignés aux femmes - et aussi aux attentes des femmes elles-mêmes. À la femme pleine d'appétit du début de l'époque moderne succède la prude du dix-neuvième siècle, évolution que recoupent les recherches sur le sort des femmes dans la sphère domestique et le monde du travail.

Un nombre croissant de recherches historiques sur les rôles sexués des femmes, la sexualité et celles, plus récentes, sur "le corps" ont contribué à produire un récit historique sur les femmes, depuis

27 Merchant $1980: 148$.

28 McLaren 1985: 324, 330 et 340. Pour des exemples de commentaires sur la montée en puissance de la femme désexualisée dans des contextes très variés, voir Accati 1990 ; Crouch 1997 : 58-78 ; Schiebinger 1987 et ead. $1996: 163$.

29 George 1973 : 152-177 ; Armstrong 1987.

30 Références incontournables : Clark Alice 1919, rééd. 1982 ; Hill 1989 ; Pinchbeck 1930, rééd. 1981 ; Davidoff \& Hall 1987. Pour un exposé critique de ces travaux, voir Vickery 1993 : 383-414. 
l'époque médiévale jusqu'à l'époque contemporaine, où le dixhuitième siècle joue un rôle pivot. De nombreuses histoires du genre intègrent, à des degrés divers, le corps dans leurs explications de la transformation qui est intervenue alors. Si certaines soulèvent des questions importantes sur le récit élaboré par Thomas Laqueur, toutes l'incorporent à leurs propres travaux. Le récit épique qu'Anthony Fletcher a donné dans Gender, sex and subordination in England, 1500-1800 (1995) est bâti sur l'idée que la période comprise entre 1660 et 1800 est un moment où se créent de "nouvelles relations de genre» au sein desquelles «les frontières entre les hommes et les femmes sont devenues plus nettes $»^{31}$. L'ouvrage débute par un passage sur le corps; Fletcher identifie des changements dans les relations de genre qui sont en partie fondés sur des changements intervenus dans la conception que l'on se faisait du corps : ce sont davantage les contrastes que la hiérarchie entre les corps qui sont alors mis en avant ${ }^{32}$. Le début du dix-septième siècle marque «la fin de l'homologie corporelle» dans les écrits médicaux, bien que la théorie des deux semences et les notions qui permettent d'établir une commensurabilité entre les sexes perdurent ${ }^{33}$. Toutefois, c'est pendant la Restauration, soit à partir des années 1660, que les auteurs commencent à concevoir les deux sexes comme incommensurables ${ }^{34}$. Anthony Fletcher prend soin de déclarer que le changement n'est pas nettement marqué ${ }^{35}$. De fait, le récit laqueurien perd ici un peu de sa vigueur : privilégiant les seizième et dix-septième siècles plutôt que le dix-huitième, Anthony Fletcher souligne que cette période constitue un «monde de transition qui n'est fait ni d'un seul sexe, ni de deux $»^{36}$. Néanmoins, la thèse selon laquelle des changements sont intervenus dans la conception que l'on se faisait du corps avant 1800 semble renforcer l'idée que le dix-huitième siècle joue bien un rôle pivot et, dans ses grandes lignes, cette narration est

\footnotetext{
$31 \quad$ Fletcher $1995: 407$.

32 Ibid. : xvi-xvii, 291 et 402.

33 Ibid. : 36.

34 Ibid. : 41.

35 Ibid. : 296.

36 Ibid. : 41.
} 
en accord presque parfait avec celle de Laqueur ${ }^{37}$. De plus, bien qu'il ne retienne pas l'explication politique de Thomas Laqueur, Anthony Fletcher avance l'idée que les changements observés résultent de la redéfinition du patriarcat par les hommes qui cherchaient à en assurer la pérennité et à en renforcer les fondements ${ }^{38}$.

Robert B. Shoemaker, dans Gender in English society, 1650-1850: the emergence of separate spheres? (1998), propose une minutieuse histoire culturelle de la vie des hommes et des femmes, livrant une synthèse importante sur les pratiques sexuées et les représentations de genre. Il décrit la "valeur croissante qui a été accordée à des rôles sexuels distincts chez les hommes et les femmes » et relie ce constat au " développement du corps à deux sexes et à l'importance idéologique croissante accordée à l'acte sexuel avec pénétration vaginale $»^{39}$. Bien que Robert Shoemaker prenne soin de souligner l'augmentation des opportunités dont pouvaient se saisir les femmes, dans le même temps, il note que le caractère régressif des idées que l'on s'en faisait était directement lié à celles qui avaient trait à leur corps. «À l'extérieur de la sphère domestique, les femmes ont pu profiter d'une palette d'opportunités plus large, écrit Robert Shoemaker, mais elles furent simultanément freinées par des critères moraux plus contraignants ainsi que par des croyances sur l'influence débilitante de leurs nerfs et de leurs fonctions biologiques $»^{40}$. Comme dans beaucoup de travaux récents, la thèse majeure de Thomas Laqueur d'une évolution du modèle unisexe vers le modèle des deux sexes, s'intègre à un tableau beaucoup plus large qui incorpore à la fois le désir sexuel et les rôles sexués.

Dans English sexualities, 1700-1800 (1997), Tim Hitchcock tente d'établir un lien entre les observations des taux de fertilité qui ont été faites par les démographes et les pratiques sexuelles. Pour lui, la révolution sexuelle a coïncidé, au tout début du dix-huitième siècle, avec une idéologie nouvelle qui établissait des sphères séparées, mais aussi avec le passage de la conception unisexe du corps humain à la

\footnotetext{
37 Ibid. : 291.

38 Ibid. : xxii.

39 Shoemaker $1998: 85$. Voir également : 31-35 et 313-314.

40 Ibid. : 34.
} 
conception des deux sexes: la conception médicale du corps des femmes qui a triomphé est celle dans laquelle «l'inégalité et les "sphères séparées" étaient inscrites dans une biologie irréfutable » ${ }^{41}$. Hitchcock parle "d'une transition entre l'époque moderne où des ouvertures et des possibilités nombreuses étaient offertes aux femmes à une situation dans laquelle [...] la capacité des femmes à participer à la sphère publique s'est progressivement réduite ». Le glissement du modèle unisexe vers le modèle des deux sexes est une composante inséparable de ce changement, de même que les transformations des idées sur le désir féminin: "Initialement perçues comme sexuellement entreprenantes, écrit Hitchcock, les femmes sont devenues sexuellement passives $»^{42}$. Bien que Tim Hitchcock souscrive au point de vue selon lequel il y a eu une "redéfinition du corps des femmes et des hommes à la fin du dix-huitième siècle », il précise néanmoins que cette redéfinition s'est déroulée sur une durée beaucoup plus longue que Laqueur ne l'admet ${ }^{43}$. Cependant, idéaux de genre, désir sexuel et comportement sexuel ont subi un changement radical :

La sexualité elle-même a changé et [...] les personnes qui pratiquent une sexualité hétérosexuelle restreignent de plus en plus leurs pratiques à des formes de rapports sexuels phallocentriques avec pénétration, au prétexte qu'elles sont procréatrices. De ce fait, les définitions de la "masculinité" et de la "féminité" changent aussi. Les hommes et les femmes ont été créés "naturellement" et biologiquement sexués, avec l'obligation croissante $[\ldots]$ de limiter leur comportement à une norme hétérosexuelle, et de trouver l'autre sexe, nouvellement appelé sexe "opposé", attirant ${ }^{44}$.

Cette évolution a conduit à définir "un ensemble "naturel" de catégories hétérosexuelles bien déterminées » qui, à son tour, restreint le nombre de comportements et d'identités pour ceux qui sont attirés par des personnes de même sexe ${ }^{45}$. Le corps est l'un des éléments d'une transformation plus importante dans laquelle des formes de

\footnotetext{
41 Hitchcock 1997 : 48. On retrouve ces arguments dans Hitchcock 1996 : 72-90.

42 Hitchcock $1996:$ 77-78.

43 Hitchcock $1997: 57$.

44 Ibid. : 111.

45 Ibidem. Voir aussi : 24-41 et 58-92.
} 
comportement sexuel et des catégories d'identité sexuelle se sont modifiées.

Les arguments de Tim Hitchcock sont en grande partie étayés par l'ouvrage de Randolph Trumbach, Sex and the Gender Revolution: Heterosexuality and the Third Gender in Enlightenment London (1998). Pour Trumbach, une révolution sexuelle a produit un troisième genre - les «nouveaux adultes sodomites efféminés »-et a introduit pour les hommes une nette distinction entre un désir exclusivement hétérosexuel et un désir exclusivement homosexuel: ces bouleversements sont étroitement associés au modèle des deux sexes. Avant 1700, il y avait "trois sortes de corps (hommes, femmes et hermaphrodites)» mais «seulement deux sortes de genres (masculin et féminin) ». Après 1700, "il y a désormais deux sortes de corps (masculin et féminin) mais trois genres (homme, femme et sodomites) $»^{46}$. Dans ce livre, et dans des articles antérieurs importants, Trumbach décrit un durcissement de la masculinité et de la féminité, la première se mesurant à l'aune des nouveaux sodomites efféminés, la seconde à l'aune de la prostituée ${ }^{47}$. Ce sont sans aucun doute les femmes pauvres qui, dans le scénario de Randolph Trumbach, sont les principales perdantes : «il est vraisemblable que ce sont elles qui ont le plus souffert de la construction du monde sexuel moderne $»^{48}$. Accouchant souvent d'enfants illégitimes, n'ayant pas accès à l'idéal de l'amour romantique ni à celui de la vie de famille que les femmes de statut supérieur entretenaient pour adoucir les mauvais traitements que les hommes leur infligeaient, souffrant de ne pouvoir endosser le rôle respectable de la femme passionnée, leur réputation était des plus précaires. En effet, tandis que le désir sexuel des hommes était essentiel à « la nouvelle hétérosexualité masculine », les femmes étaient souvent punies quand elles prenaient l'initiative, et seules les prostituées étaient censées afficher «un désir sexuel exceptionnel $»^{49}$. Selon Randolph Trumbach en effet, après la révolution sexuelle, les seules femmes qui purent exprimer une

\footnotetext{
46 Trumbach $1998: 9$.

47 Trumbach $1991:$ 186-203, et 1989.

48 Trumbach $1998: 322$.

49 Ibid : 394 et 424.
} 
passion sexuelle étaient les prostituées, les domestiques séduites, les veuves remariées ou les femmes adultères.

Le modèle des deux sexes, la désexualisation des femmes et la séparation des sphères apparaissent invariablement comme des évolutions concomitantes qui ont appauvri qualitativement la place des femmes. Comme l'écrit Janice F. Thaddeus :

Au début du dix-huitième siècle et auparavant, les femmes sont censées ressembler aux hommes. Même leur corps - bien que moins parfait, bien sûr - est censé ressembler à celui des hommes. De là, l'idée que les femmes sont fortes et sensuelles, qu'elles sont presque aussi indépendantes après le mariage qu'avant. En 1788, cet être féminin, défini essentiellement comme une version amoindrie de l'homme, a été redéfini comme un être séparé et opposé à l'homme, chaste et domestique par "nature" 50 .

De la même manière, pour Angus McLaren, le changement dans la conception de la sexualité féminine a mis fin à cette "situation assez égalitaire » dans laquelle «le lit était un endroit où hommes et femmes étaient plus ou moins égaux». Plus encore, «le droit des femmes au plaisir sexuel n'a pas été valorisé, mais déprécié $»^{51}$. On trouve cette même idée dans certaines reformulations récentes des thèses sur la restriction des opportunités de travail pour les femmes ; ici, le déclin de l'économie familiale aurait altéré « une situation qui ressemblait à un partenariat de travail établi avec leurs maris » et qui se serait apparentée à une " égalité réelle ${ }^{52}$. Tim Hitchcock indique que les changements observables en matière de sexualité et d'identité «ont annoncé une période d'intense oppression patriarcale. Mais, pour les hommes également, cela s'est traduit par une conception de plus en plus contraignante de la masculinité $»^{53}$. Robert Shoemaker s'oppose avec vigueur à ce récit pessimiste, faisant valoir, qu'en dehors de la sphère domestique, les possibilités laissées aux femmes ont augmenté, malgré les images "de plus en plus contraignantes » véhiculées par les manuels de bonne conduite et la littérature ${ }^{54}$.

\footnotetext{
50 Thaddeus $1994: 113$.

51 McLaren $1985:$ 340-341.

52 Hill 1989 : 46. Voir aussi Snell $1981:$ 407-437.

53 Hitchcock $1997: 111$.

54 Shoemaker $1998: 42$.
} 
Néanmoins, Shoemaker suggère que les images des femmes diffusées par la culture déterminent de plus en plus la vie des individus, et observe que « les rôles de genre de la période moderne étaient moins intériorisés et moins contraignants qu'ils ne le sont devenus à l'époque victorienne $»^{55}$. Il conclut qu'une «accentuation» a eu lieu dans la construction et la pratique de la séparation sexuée - la constitution de sphères séparées - entre 1650 et $1850^{56}$. De telles interprétations soulignent qu'il y a non seulement eu des changements dans la conception des rôles et des idéaux, mais aussi que la nature même de ces idéaux a été marquée par une plus grande rigidité et le rétrécissement des perspectives. De fait, des argumentations antérieures suivant lesquelles les producteurs d'image «définissaient les relations homme/femme avec une précision nouvelle» et les femmes enduraient «la longue marche des Empires du genre sur l'entièreté de la personne» ont trouvé un écho dans l'hypothèse récente qu'« une rigueur plus importante a été imposée à la définition des rôles de genre $»^{57}$. Tim Hitchcock et Michèle Cohen écrivent, dans leur introduction à la collection English masculinities, 1660-1800 (1999) : «l'ancien modèle d'un renforcement progressif des frontières $\mathrm{du}$ genre est actuellement remis en question $»^{58}$. Cependant, cette position n'a pas encore été étayée sur des travaux publiés et les études antérieures qui ont montré de quelle façon la conception de la masculinité s'était restreinte n'ont pas encore été revisitées ${ }^{59}$. L'attention récente que George Haggerty porte à l'amour entre hommes situe plutôt ces relations dans une culture «qui apprenait tout juste à codifier la différence sexuée et à marginaliser les comportements excessifs; à construire une sexualité comme un système binaire rigide destiné à isoler le "non-naturel"; et à construire une identité en enfonçant un coin entre le discours public et le discours privé ${ }^{60}$. Dans la vision qui est ici proposée du

\footnotetext{
55 Ibid. : 317.

56 Ibid. : 318

57 George 1973 : 159; Riley 1988 : 14 ; Hitchcock 1996 : 77.

58 Cohen \& Hichcock 1999 : 18 (Introduction).

59 Voir, par exemple, Senelick 1990 : 33-67. Pour un exemple équivalent en Europe, voir Liliequist 1991 : 393-423.

60 Haggerty $1999: 172$.
} 
changement, les individus ont été de plus en plus saturés de notions ayant trait à la différence de genre et le tableau de la période antérieure au dix-huitième siècle apparaît comme un âge d'or de liberté pour les identités de sexe et de genre.

L'historiographie du corps et de la sexualité a apporté une contribution majeure à l'histoire des femmes et du genre en permettant d'historiciser les catégories et les identités sexuelles. Le constructionnisme social, qui se situe en opposition apparente à une approche biologique essentialiste, a ainsi joué un rôle important dans ces recherches. Comme l'a récemment précisé un chercheur, le constructivisme social «a produit, comme par magie, de nombreuses sexualités là où auparavant il n'y en avait qu'une $\aleph^{61}$. L'héritage légué par Michel Foucault est également fondamental. Foucault a donné la priorité à l'efficience (agency) du discours et a établi que la sexualité était le « corrélatif de cette pratique discursive lentement développée » qu'est la science du sexe ${ }^{62}$. Dans son Histoire de la sexualité, il décrit le corps comme le produit d'une pratique discursive, comme un écran sur lequel le pouvoir non-discursif projette des effets ${ }^{63}$. Aujourd'hui, il faut aussi tenir compte de l'influence de Judith Butler, qui écrit que le genre est «l'ensemble des moyens discursifs/culturels par quoi "la nature sexuée" ou un "sexe naturel" est produit et établi dans un domaine "prédiscursif", qui précède la culture » ${ }^{64}$. Cette thèse - selon laquelle les éléments qui apparaissent comme des distinctions naturelles ou biologiques sont en fait les produits de la culture et du langage - peut être facilement calquée sur l'argument de Laqueur, qui soutient que la différence sexuelle a été "fabriquée » au dix-huitième siècle. De plus en plus, les recherches sur le corps et la sexualité doivent beaucoup à une approche qu'on pourrait schématiquement

61 Milligan $1993: 109$.

62 Foucault 1976: 91 (en anglais 1979: 68). Sur Foucault et le discours, voir Thacker 1997 ; McNay 1992.

63 Sur l'approche générale du corps de Foucault, voir McNay 1992: 28 et 38-40.

64 Butler 2006 : 69 (en anglais 1990 : 7). Voir également ead. 2009 (édition originale 1993). 
qualifier de "poststructuraliste», dans laquelle la connaissance du passé vient "non pas de la reconstruction des expériences "objectives" mais d'une analyse des systèmes de signification qui, d'entrée de jeu, rendent possibles et construisent ces expériences $»^{65}$. L'impact en est particulièrement perceptible dans les discussions sur l'homosexualité d'antan, où une question centrale demeure, celle de savoir si les historiens peuvent appréhender le comportement et l'identité homosexuels à des époques où ni le concept d'homosexualité - ni sa nomenclature-n'avaient encore été inventés ${ }^{66}$.

Le fait que l'accent soit mis sur le discours a comme conséquence que l'histoire qui en découle est mieux outillée pour produire une description qu'une explication. Comme l'écrit Trumbach à la fin de l'introduction de son ouvrage récent :

Il semble que nous ne sachions pas très bien pourquoi ou comment les systèmes culturels changent si rapidement au cours d'une seule et même génération, que l'on soit en 1700 ou en 1960. C'est aussi déroutant que d'essayer d'expliquer l'expansion et la régression des maladies - peste, syphilis, variole, SIDA. C'est pourquoi, dans ce livre, nous nous contenterons d'aborder l'analyse de la division du monde sexuel en une minorité homosexuelle et une majorité hétérosexuelle, division qui reste un des aspects les plus fondamentaux de la culture occidentale moderne telle qu'elle a été constituée par la génération de $1700^{67}$.

D'autres chercheurs qui travaillent sur le discours ont des difficultés à fournir des modèles de causalité. Tout en essayant de revenir «aux racines de l'analyse de genre dans l'histoire sociologique », Shoemaker reconnaît «les progrès théoriques de ces dernières années et l'importance du langage dans la construction des rôles de genre », ce qui l'oblige à tenir compte du « rôle des idées et

65 Newman 1991: 62. Pour d'autres discussions utiles voir Riley 1988 ; Bennett 1989 : 251-272 ; Hall 1991 : 204-210 ; Kent 1996 : 9-18 ; Purvis 1996 : 5-7.

66 Voir en particulier Weeks 1989, qui soutient la thèse selon laquelle l'identité homosexuelle masculine n'est apparue qu'à la fin du XIXe siècle. Trumbach soutient au contraire que c'est au début du XVIII siècle qu'une sous-culture homosexuelle moderne a vu le jour. Voir Trumbach 1989 et 1991. Pour un exposé sur l'homosexualité avant le XVIII" siècle, voir Bray 1982.

67 Trumbach $1998: 22$. 
des idéologies» dans l'expérience sexuée. Cependant, son travail cherche "principalement à consigner les différences plutôt qu'à les expliquer ${ }^{68}$. L'attention particulière accordée au langage dans l'histoire de la sexualité et du genre, et de manière générale dans l'histoire culturelle, produit des études qui sont mieux équipées pour analyser la synchronie que la diachronie, mais aussi le sens que la causalité.

Face aux difficultés rencontrées pour expliquer le changement, on a fait appel à des cadres explicatifs plus anciens, qui ont comblé les vides creusés par l'attention nouvelle portée à la culture. Même s'il critique le seul recours au réductionnisme économique, Tim Hitchcock écrit «que le changement économique et social peut expliquer la transformation de la culture sexuelle anglaise $»^{69}$. Il suggère provisoirement que la croissance économique, l'industrialisation et l'urbanisation ont changé la culture sexuelle à travers deux mécanismes: la culture populaire de l'imprimé; la philanthropie urbaine et la politique sociale. La culture de l'imprimé a remis en question l'autorité que détenaient les personnes âgées et les femmes en particulier, en mettant à la portée des individus de nouvelles sources du savoir sur la sexualité. De plus, une politique sociale a été instaurée pour orienter l'attribution des ressources aux paroisses urbaines en expansion, aux nouvelles institutions urbaines, aux hôpitaux caritatifs, et aux asiles. Cette politique sociale a construit une féminité qui a partie liée avec la notion de victime et une masculinité définie comme sexuellement irresponsable. Tandis qu' « un amalgame complexe de forces économiques et culturelles » a joué un rôle dans la révolution sexuelle du dix-huitième siècle, Hitchcock nous met en garde en précisant que ces deux mécanismes «ne remplacent pas le rôle plus large joué par le changement économique dans l'explication de la transformation historique $»^{70}$. Le cadre explicatif tracé par Anthony Fletcher accorde plus d'efficience aux facteurs culturels : «À l'origine, il y avait le besoin des hommes

\footnotetext{
68 Shoemaker $1998: 5$ et 309.

69 Hitchcock $1997: 112$.

70 Ibid. : 112-14. Citation : 114.
} 
de consolider les fondements et l'avenir du patriarcat $»^{71}$. Leur réussite fut «d'adapter et de transformer le patriarcat en Angleterre en remplaçant sa base scripturaire et médicale ancienne [...] par une nouvelle idéologie séculière du genre »; le programme consistait à inculquer une conception «de la différence sexuelle qui soit fondamentale et incontournable $\gg^{72}$. Initialement, c'est le bouleversement des systèmes de pensée qui entraîna une diminution de l'importance et de la signification de la «Grande Chaine de l'Existence, du macrocosme et du microcosme, de la médecine humorale et de l'astrologie»; ce bouleversement fut "la cause de l'effondrement du genre traditionnel et le signal de la quête d'un nouveau cadre pour les relations de genre $\gg^{73}$. Le patriarcat fut réinventé alors qu'il menaçait de perdre de sa stabilité dans un contexte idéologique en pleine mutation.

Ces travaux se calquent sur des récits économiques et politiques reconnus et, en outre, ils ont servi à donner un second souffle à des modèles de changement plus anciens en absorbant le scénario laqueurien. Pourtant certaines objections ont été faites à ce scénario tel qu'il a été présenté dans des histoires récentes du genre. Certains travaux d'historiens sur des époques antérieures et postérieures mettent en doute l'affirmation selon laquelle le dix-huitième siècle a constitué un tel tournant. Les historiens des seizième et dix-septième siècles avancent l'idée que des changements importants dans la compréhension du genre et du corps ont commencé avant $1700^{74}$. Mary Fissell décrit la manière dont les ouvrages médicaux en langue vernaculaire de la deuxième moitié du dix-septième siècle remettent en question l'hégémonie du modèle unisexe $\mathrm{e}^{75}$. Ce travail, qui porte sur la période antérieure à 1700, suggère que l'accent est mis sur la différence des corps bien plus tôt que Laqueur ne l'admet. De plus, depuis quelque temps, les historiens qui travaillent sur le dix-

\footnotetext{
71 Fletcher 1995 : 401.

72 Ibid: 283 et 407.

73 Ibid. : 407.

74 Crawford \& Mendelson 1998 : 29-30 ; voir également l'exposé de Fletcher plus haut, $1995:$ xxx.

75 Fissell $1995: 433-456$.
} 
neuvième siècle remettent en question le stéréotype de la femme victorienne asexuée, tandis que d'autres font remarquer que la sexualité masculine était également soumise à des règles ${ }^{76}$. Plus récemment, on a pu écrire que, dans sa façon d'appréhender le clitoris, la profession médicale victorienne a renforcé «l'hétérosexualité et les frontières du genre, plutôt qu'elle ne s'est engagée dans une répression de la sexualité féminine ${ }^{77}$. Une telle affirmation n'est pas sans rappeler la caractérisation du dix-huitième siècle qui est faite par Trumbach et Hitchcock. Le caractère unique du dix-huitième siècle est également remis en question par ceux qui, dans le passé, ont soutenu que la science du dix-neuvième siècle a produit un schéma des différences sexuelles qui tentait de museler l'aspiration des femmes à participer à la vie politique au-delà de la sphère domestique $^{78}$. Si un changement de fond s'est produit dans la façon dont la science et la médecine représentent le corps - changement motivé par un besoin de concentrer le pouvoir entre les mains de quelques-uns -, il n'est clairement pas achevé à la fin du dix-huitième siècle. Il est probable que toute cette transformation a suivi un très long processus.

De plus en plus de voix discordantes revendiquent une autre approche du changement dans l'histoire du corps, de la sexualité et du genre. Il semble en effet que les modèles de changement établis pour une sphère donnée ne soient pas toujours transposables à une autre. Certains historiens pensent que les récits pertinents de changement ne sont valides que s'ils sont établis sur une période courte correspondant au cycle de vie d'un individu et non à l'échelle, beaucoup plus grande, des transformations historiques. Sara Mendelson et Patricia Crawford ont intégré leurs études sur le sexe, la contraception, la grossesse et l'accouchement à une recherche sur la vie des femmes, de l'enfance à l'adolescence et à l'âge adulte ${ }^{79}$. La notion de "cycle de vie" est précieuse également pour faire l'histoire

\footnotetext{
76 À propos de la sexualité féminine voir, par exemple, Nead 1988 ; Peterson 1986 : 569-590. Sur la sexualité masculine, voir Hall \& Porter 1995 : 141-145.

77 Moscucci $1996: 72$.

78 Alaya $1977: 264$; Russett $1989: 2$ et 10.

79 Crawford \& Mendelson 1998 : 118-122 et 148-156.
} 
de la masculinité. Les recherches de Tim Hitchcock sur la vie sexuelle de John Cannon montrent que les années qui suivent ses vingt ans jusqu'au début de sa trentaine sont des années cruciales de changement dans ses pratiques sexuelles ${ }^{80}$. En plus de cette attention portée au cycle de vie, les spécialistes prennent soin de rappeler que les continuités sont tout aussi présentes que les changements dans l'histoire du corps et de la sexualité ${ }^{81}$. Dans la littérature érotique, par exemple, les conceptions de la différence sexuelle ne peuvent être facilement emboîtées dans les modélisations spécifiques à chaque période. Au contraire, deux façons d'envisager la différence des sexes ont coexisté tout au long du dix-huitième siècle ${ }^{82}$. Chez les historiens des femmes et du genre, cet accent mis sur la durée dans l'histoire du corps participe d'un intérêt plus général tant pour la permanence que pour la transformation ${ }^{83}$.

L'intégration des arguments sur les cycles de vie et la permanence historique dans l'histoire du corps, de la sexualité et du genre modifie nécessairement les récits qui privilégient la longue durée et le caractère linéaire du changement. Les études sur les échanges culturels et sur la place des textes au sein de la société sont venues infléchir encore davantage le discours dominant. L'affirmation selon laquelle le corps, le genre et la sexualité sont redéfinis en fonction des débats sur la représentation politique tend à subsumer toute une série de phénomènes historiques sous le seul débat politique. La même remarque s'applique à l'utilisation des sources: des types d'informations différentes sont issus de documents de natures et aux fonctions diverses. Pour étayer l'affirmation selon laquelle, au même moment, les romans, les sermons et autres écrits didactiques, les pièces de théâtre, les dossiers judiciaires, les traités de philosophie, les traités scientifiques et les livres de médecine ont tous été traversés par les motivations des penseurs politiques, il faudrait pouvoir établir des

\footnotetext{
80 Hitchcock $1999: 25-43$ et $1997: 28-38$.

81 Gowing 1996 : 28-29 ; Crawford \& Mendelson 1998 : 29-30 ; Shoemaker 1998 : 20-21, 61 et 86.

82 Voir Harvey 2002 : 202-223.

83 Tosh 1999 : 238 ; Gowing 1996 : 28-29.
} 
liens entre ces sources et des réseaux de communication plus larges ${ }^{84}$. Les théories qui circulaient dans les textes médicaux ont pu influencer les ouvrages littéraires qui traitent de la différence sexuelle et du corps mais, en retour, ces théories s'en sont trouvées modifiées, elles aussi. Par exemple, à la fin du dix-septième siècle, l'ouvrage de Mauriceau destiné aux sages-femmes, Les maladies des femmes grosses, se révèle riche d'enseignements pour les auteurs de la littérature érotique, qui utilisent des métaphores botaniques et cartographiques pour décrire le corps humain. Mais, tout en s'inspirant largement des descriptions que fait Mauriceau de la conception humaine, ces auteurs participent aux discussions sur ses théories et les utilisent de manière sélective $\mathrm{e}^{85}$. Les écrits médicaux et érotiques sont liés par leur intérêt commun pour certains thèmes, et même pour certains mots et certaines images, mais ils ne disent pas la même chose. En effet, un genre littéraire - et même un texte isolé - est rarement univoque et une seule lecture n'en épuise pas le sens. Les ouvrages scientifiques et médicaux spécialisés contiennent de multiples points de vue sur la différence sexuelle. Réexaminant les images contenues dans les textes médicaux de la période moderne, Robert Martensen conclut qu'ils présentent alternativement le modèle d'un sexe unique et le modèle des deux sexes ${ }^{86}$. L'ouvrage populaire de médecine Aristotle's compleat master-piece déploie sans aucun doute les deux discours : on y déclare sans équivoque que «les femmes ne sont rien d'autre que des

84 Un bon exemple de ce type de recherche est le travail de Robert Darnton sur les ouvrages interdits en France à l'époque de la Révolution. Voir Darnton 1995b : 181-197.

85 Francis Mauriceau, The diseases of women with child: as also the best means of helping them in natural and unnatural labours, (7e éd., trad. Hugh Chamberlain, 1736). Traduction de l'ouvrage français: François Mauriceau, Des Maladies des femmes grosses et accouchées avec la bonne et véritable méthode de les bien aider..., Paris, J. Hénault, 1668. Le plagiaire érotique est Philogynes Clitorides [pseud., alias Thomas Stretser ?], The natural bistory of the frutex vulvaria, or flowering shrub ([c. 1737]; Arbor vitae: or, the natural history of the tree of life, 1741). Voir Harvey $2002: 203-206$.

86 Martensen 1994 : 107-133. 
hommes retournés comme un gant», tout en informant les lecteurs que « la différence des sexes est un pré-requis à la conception $»^{87}$.

Le travail de Laqueur a été critiqué non seulement parce qu'il a présenté «une interprétation trop schématique et simpliste des changements de la théorie médicale », mais aussi parce qu'il «n’a pas pris en compte les informations non-médicales issues de la compréhension populaire de la conception et de la différence sexuelle » telles qu'on les trouve dans la pornographie, les traités de vulgarisation médicale et les dépositions judiciaires ${ }^{88}$. La culture n'étant pas monolithique, une telle variété d'informations produit différents types de conceptions du corps. Les corps mis en scène dans la pornographie, au lieu d'être présentés comme opposés, fortement sexués et incomparables, peuvent être décrits comme «aussi semblables, égaux et dénués de genre que les atomes et les planètes $»^{89}$. Dans certains ouvrages meilleur marché, la conception populaire de la sexualité "dévoie » les discours médicaux tandis que dans la culture féminine orale ou écrite, on trouve des mises en doute des théories qui ont cours chez les élites ${ }^{90}$. Si l'on a peu de travaux sur la façon dont les hommes vivaient leur corps et sur la manière dont ils percevaient la différence sexuelle, Elizabeth Foyster soutient malgré tout que, "par le passé, la revendication de la "différence" était aussi répandue chez les hommes que chez les femmes ${ }^{91}$. Comme l'a démontré Laura Gowing à travers des pièces de procès qui ont eu lieu entre 1560 et 1640, les hommes et les femmes ordinaires avaient tendance à mettre plutôt l'accent sur la différence que sur la similitude entre les sexes. Le rôle central de l'orgasme des femmes dans la conception était chose communément admise mais l'idée selon laquelle « la femme et l'homme auraient été deux moitiés d'un même sexe ne semble pas avoir pris racine dans l'imagination

87 Aristotle's compleat master-piece: in three part: displaying the secrets of nature in the generation of man (11e éd. [1725], p. 14 et 30). À propos des différentes éditions de ce manuel populaire, voir Porter 1987 : 1-21.

88 Jenner \& Taithe $2000: 194$.

89 Voir Jacob $1993: 182$.

90 Crawford $1994: 82-106$.

91 Foyster $1999: 29$. 
populaire $»^{92}$. Dans le langage courant, des termes assez différents étaient utilisés pour désigner les organes génitaux masculins et féminins, et cela à une époque où les écrits scientifiques et médicaux n'avaient pas encore mis au point une nomenclature distincte pour le corps féminin ${ }^{93}$. Un langage familier qui distingue ces parties du corps suggère que le corps féminin et le corps masculin étaient considérés comme structurellement, fonctionnellement et moralement distincts. On pouvait très bien faire référence à certaines parties du modèle unisexe sans pour autant adhérer à la vision d'ensemble.

Le «sentiment» qui fait dire à Laqueur que "médecins, auteurs profanes, ainsi qu'hommes et femmes, dans leur couche, partageaient grosso modo la même idée du fonctionnement du corps en matière de reproduction $»^{94}$ ne semble pas devoir être suivi. La lecture croisée de la pornographie, des traités de vulgarisation médicale et des archives judiciaires révèle toute une gamme de conceptions de la relation homme-femme. Plutôt que d'établir une distinction entre une conception « populaire » et une conception « élitaire » du corps, cette variété de conceptions met l'historien au défi de déchiffrer quand, où, et pourquoi on a utilisé des modèles différents ${ }^{95}$. À la suite de Ludmilla Jordanova qui a inspiré les historiens de la culture, les historiens du corps et de la sexualité pourraient se demander en quoi le contenu et la forme des sources historiques «médiatisent les conditions sociales où elles prennent place $»^{96}$. En d'autres termes, quelles fonctions remplissent les concepts de «différence» et de «similitude» corporelles dans les diverses occurrences où on les rencontre et comment sont-elles utilisées ? Bien qu'elle mette l'accent sur la différence entre les hommes et les femmes ordinaires, Laura Gowing pense aussi que l'idée de similitude corporelle a pu servir «à donner un sens à la sexualité : les corps de femmes et d'hommes ne

\footnotetext{
92 Gowing $1996: 7$.

93 Ibid. : 80-82 ; Laqueur 1992 : 123-125 (en anglais : 96-98).

94 Laqueur $1990: 68$.

95 Tim Hitchcock fait une distinction entre ce qui est "populaire » et " élitaire », mais aucune référence à des sources (prix, lectorat, marketing) ne vient valider cette distinction. Voir Hitchcock $1997: 54$.

96 Jordanova $1999: 3$.
} 
s'emboîtent pas seulement sur le papier, mais aussi en pratique $»^{97}$. Dans la littérature érotique du dix-huitième siècle, les auteurs utilisaient des homologies quand ils cherchaient à faire comprendre qu'il était question de pénétration vaginale ou de fertilisation. La différence pouvait alors être accentuée mais, dans le même temps, la similitude corporelle facilitait la compréhension de l'union des hommes et des femmes ${ }^{98}$.

Du fait de cet intérêt pour le discursif, les contextes concrets de la sexualité sont restés largement inexplorés et certains historiens regrettent que cette focalisation ait exclu les corps physiques des documents historiques. Lyndal Roper déplore que «le corps» dans le travail historique soit "une irritante abstraction non-physique » et soutient, qu'au contraire, "nous avons besoin d'une compréhension de la différence sexuelle qui intègre le corporel au lieu de le combattre ${ }^{99}$. La plupart des travaux existants mettent l'accent sur les changements intervenus dans le langage et le discours mais semblent rejeter les continuités établies par le corps physique. L'ouvrage de Sara Mendelson et Patricia Crawford fait exception et met l'accent sur les expériences typiquement féminines que sont la menstruation, la parturition, la lactation, expériences qui fondent très durablement l'insistance qui est mise, au plan culturel, sur l'infériorité du corps féminin ${ }^{100}$. Une autre direction de recherche pour l'étude de la sexualité reste encore très inexploitée : celle de la production et de la consommation d'ouvrages se rapportant au sexe, au désir et au corps. De nombreux travaux d'histoire de la sexualité considèrent que ces ouvrages suscitaient des réactions prévisibles chez les contemporains et qu'ils peuvent être utilisés comme des indicateurs des expériences que les gens ont pu vivre. Hitchcock reconnaît qu'il est difficile de savoir à quel point les individus ont été affectés par ces écrits mais il souligne que « la nature du cadre idéologique dans lequel les femmes agissaient était changeante, et que les attentes contenues dans les guides sur la sexualité et les manuels de bonne conduite étaient de

\footnotetext{
97 Gowing $1996: 7$.

98 Harvey 2002.

99 Roper 1994 : 17-18.

100 Crawford \& Mendelson $1998: 18-30$.
} 
plus en plus contraignantes ». Dans son étude des représentations des rôles de genre, Tim Hitchcock indique que «c'est aux femmes qu'on imposait le plus grand refoulement $»^{101}$. Shoemaker commence par envisager «les rôles de genre vécus par chacun comme le produit de l'interaction entre des façons de penser le genre et une combinaison de forces sociales, économiques et politiques $\gg^{102}$. Mais, en définitive, il semble douter de l'impact de l'idéologie sur la construction des rôles de genre : il montre la réduction des représentations et, malgré tout, un accroissement des opportunités ${ }^{103}$. Ce qui manque donc le plus à l'ouvrage, c'est une explication des réactions des individus (et de leur résistance, dont il est question dans le livre) face à des idées de plus en plus étriquées.

Ce qui fait certainement le plus défaut dans l'historiographie du corps et de la sexualité est une recherche qui tenterait d'évaluer jusqu'à quel point la vision de Laqueur correspond à l'expérience des hommes et des femmes. Comme John Tosh l'a fait remarquer, "cela vaut la peine de se demander si la polarisation sexuelle n'a pas été qu'une figure de rhétorique. Est-ce que cela reflétait la réalité des relations entre les hommes et les femmes ? „104. Tim Hitchcock et d'autres ont assurément soulevé la question du rapport entre les publications émanant des élites, les conceptions populaires et les expériences des hommes et des femmes ${ }^{105}$. Mais, face à une absence quasi complète de recherche sur la question de la réception, on a le sentiment que les hommes et les femmes ne sont que des éponges ${ }^{106}$. En multipliant les recherches sur la production, la lecture et la réception, on parviendrait probablement à une remise en cause fondamentale de l'interprétation que les historiens font de leurs

101 Hitchcock $1996: 77$ et 78 .

102 Shoemaker $1998: 5$.

103 Ibid. : 11.

104 Tosh 1999 : 227.

105 Hitchcock 1997 : 49 ; Shoemaker 1998 : 60 ; Tosh 1999 : 227.

106 Anna Clark se démarque en proposant une lecture de l'identité de la lesbienne Anne Lister. Elle identifie les représentations culturelles comme l'une seulement des trois ressources mobilisées dans le passé par les individus pour construire leur identité sexuelle, les deux autres étant «leurs tempéraments et pulsions » et « leurs conditions matérielles ». Voir Clark Anna 1996 : 23-50. Citations : 27. 
données. Par exemple, Dorelies Kraakman soutient que la littérature érotique constitue une source utile «à l'historien de la sexualité, en particulier l'historien de la sexualité féminine», mais les travaux sur les conditions de production et de circulation de ces sources suggèrent que leur utilisation est plus appropriée pour l'étude de l'histoire de la masculinité107. Tandis qu'une grande partie des écrits sur le sexe à l'époque moderne semble surtout préoccupée par le corps féminin (intérêt que l'on retrouve dans « une histoire du corps » qui n'a pas grand-chose à dire sur les hommes), les préoccupations qui s'y font jour ont un rapport beaucoup plus direct avec les débats qui leur sont contemporains sur le corps et la sexualité des hommes. Si Laqueur écrit: "probablement est-il impossible d'écrire une histoire du corps de l'homme et de ses plaisirs parce que les archives historiques ont été créées dans une tradition culturelle où cette histoire n'était pas nécessaire », il n'est pas juste de dire que «c'est toujours la sexualité de la femme qui est constituée »108. De plus en plus, il semble qu'une grande partie de l'historiographie du corps et de la sexualité soit une historiographie de la masculinité et que l'histoire de la sexualité féminine reste encore à écrire.

Quand un nouveau champ de recherche s'ouvre, il y a espoir de corriger les théories anciennes et de modifier les débats en cours. Dans La Fabrique du sexe, Laqueur a démontré que des catégories apparemment naturelles sont en fait spécifiques à la culture et à l'histoire, et a apporté à l'histoire du corps et de la sexualité un récit diachronique construit sur des thèses plus anciennes. L'ouvrage place l'histoire du corps sur la carte de l'historiographie et de nombreux historiens des femmes et du genre l'utilisent comme un ouvrage représentatif de l'histoire du corps. Et, pourtant, on voit surgir de

\footnotetext{
107 Kraakman 1994 : 547. Pour une des rares analyses de la pornographie française, voir Goulemot 1991 : 29-47 (en anglais 1994 : 13-29). Voir également Darnton 1995b : 217-231. Pour le lectorat de la littérature érotique anglaise, voir Harvey 2004, chap. 2.

108 Laqueur 1992 : 38-39 (en anglais : 22).
} 
plus en plus de questions sur la place de ce travail dans le champ de l'histoire des femmes et du genre, qui reprennent d'anciennes questions portant sur l'interprétation des sources, sur les limites de ce que les historiens peuvent raisonnablement défendre et, surtout, sur le changement. La diversité synchronique des conceptions du corps mise à jour par des recherches récentes, couplée à l'évidence de la permanence, vient miner le scénario dominant de l'histoire du corps et de la différence sexuelle. Les corps ne peuvent jamais être des incarnations de la seule modernité mais, en même temps, ils en constituent l'incarnation la plus durable. De plus, l'intégration harmonieuse de la thèse de Thomas Laqueur à des travaux récents de l'histoire du genre tend à éclipser la façon dont certains domaines de la vie - le travail, l'organisation de la vie de famille, la reproduction, le plaisir - sont soumis à des forces distinctes de changement et de permanence. Les historiens du genre doivent étudier ces tensions. De plus, les incertitudes sur la chronologie ou sur les sources laissent penser que le récit politique sous-jacent à la thèse laqueurienne ne constitue pas une explication satisfaisante pour une approche culturelle globale des corps, des sexualités et du genre. Si l'histoire des femmes a constitué antérieurement à celui-ci, un récit dont la chronologie - calquée sur l'histoire économique - était imparfaite, il semblerait qu'un récit fondé sur l'évolution des débats sur la représentation politique soit tout aussi imparfait. Les historiens du genre continuent d'être mis au défi d'élaborer des modèles pertinents et des cadres explicatifs qui permettent de comprendre les changements et les permanences.

Traduction Sébastien de Villèle, avec l'aide de Florence Cabaret (Eriac, Université de Rouen)

\section{Bibliographie}

ACCATI Luisa, 1990, "The spirit of fornication: virtue of the soul and virtue of the body in Friuli, 1600-1800 », in Edward Muir \& Guido RugGiERO (eds), Sex and gender in historical perspective, Baltimore, John Hopkins University Press, p. 110-131.

AlAyA Flavia, 1977, "Victorian science and the "genus" of woman ", Journal of the History of Ideas, vol. 38, 2, p. 261-280. 
Armstrong Nancy, 1987, Desire and domestic fiction: a political history of the novel, Oxford, Oxford University Press.

BENNETT Judith, 1989, « Feminism and history », Gender and History, 1, p. 251-272.

BrAY Alan, 1982 (rééd. 1985), Homosexuality in Renaissance England, New York, Columbia University Press.

Bullough Vern L., 1993, "Medieval medical and scientific views of women", Viator, 4, p. 485-501.

BUTLER Judith, 1990, Gender trouble: feminism and the subversion of identity, New York, Routledge. Traduction française: 2006, Trouble dans le genre: le féminisme et la subversion de l'identité, traduit par Cynthia Kraus, Paris, La Découverte.

-, 1993, Bodies that matter: on the discursive limits of sex, New York, Routledge. Traduction française : 2009, Ces corps qui comptent: de la matérialité et des limites discursives du sexe, traduit par Charlotte Nordmann, Paris, Amsterdam.

CHARTIER Roger, 1993, «Book markets and reading in France at the end of the Old Regime ", in Carol Armbruster (ed.), Publishing and readership in Revolutionary France and America, Westport, CT, Greenwood Press, p. 117-136.

Clark Alice, 1919 (rééd. 1982), Working life of women in the seventeenth century, Londres, Routledge.

Clark Anna, 1996, "Anne Lister's construction of lesbian identity », Journal of the History of Sexuality, vol. 7, 1, p. 23-50.

CLARK J.C.D., 1985, English society, 1688-1832: ideology, social structure and political practice during the Ancien Régime, Cambridge, Cambridge University Press. La seconde édition a été publiée sous le titre English society, 1660-1832 (Cambridge, 2000).

CoHen Michèle \& Tim HichCock (eds), 1999, English masculinities, 1660-1800, Londres, Longman.

COT'T Nancy, 1978, « Passionlessness: an interpretation of Victorian sexual ideology, 1790-1850», Signs, 4, p. 219-236.

CRAWFORD Patricia, 1994, "Sexual knowledge in England, 1500-1750», in Roy PORTER \& Mikulás TEICH (eds), Sexual knowledge, sexual science: the history of attitudes to sexuality, Cambridge, Cambridge University Press, p. 82-108.

Crawford Patricia \& Sara MENDElSON, 1998, Women in early modern England, 15501720, Oxford, Oxford University Press.

Crouch Kimberly, 1997, "The public life of actresses: prostitutes or ladies? ", in Hannah BARKER \& Elaine CHALUS (eds), Gender in eighteenth-century England: roles, representations and responsibilities, Londres, Longman, p. 58-78.

DARNTON Robert, 1995a, The corpus of clandestine literature in France, 1769-1789, New York, W. W. Norton.

—, 1995b, The forbidden best-sellers of pre-Revolutionary France, New York, W. W. Norton.

DAVIDOFF Leonore \& Catherine HALL, 1987, Family fortunes: men and women of the English middle class, 1780-1850, Londres, Hutchinson. 
FISSELL Mary, 1995, «Gender and generation: representing reproduction in early modern England », Gender and History, 7, p. 433-456.

FLeTCHer Anthony, 1995, Gender, sex and subordination in England, 1500-1800, New Haven, Yale University Press.

Foucault Michel, 1976, Histoire de la sexualité. 1: La Volonté de savoir, Paris, Gallimard. Traduction anglaise: 1977, The history of sexuality, 1: An introduction, Victoria, Penguin Books.

FOYSTER Elizabeth, 1999, Manhood in early modern England: honour, sex and marriage, Londres, Longman.

GEORGE Margaret, 1973, "From "goodwife" to "mistress": the transformation of the female in bourgeois culture », Science and Society, 37, p. 152-177.

Goulemot Jean-Marie, 1991, Ces livres qu'on ne lit que d'une main: lecture et lecteurs de livres pornographiques au XVIII e siècle, Aix-en-Provence, Alinéa. Traduction anglaise: 1994, Forbidden texts: erotic literature and its readers in eighteenth-century France, traduction James Simpson, Cambridge, Cambridge University Press.

GowING Laura, 1996, Domestic dangers: women, words and sex in early modern London, Oxford, Clarendon Press.

HagGerTy George E., 1999, Men in love: masculinity and sexuality in the eighteenth century, New York, Columbia University Press.

HaLl Catherine, 1991, "Politics, post-structuralism and feminist history », Gender and History, 3, p. 204-210.

HALL Lesley \& Roy PORTER (eds), 1995, The facts of life: the creation of sexual knowledge in Britain, 1650-1950, New Haven, Yale University Press.

HARVEY Karen, 2002, "The substance of sexual difference: change and persistence in representations of the body in eighteenth-century England», Gender and History, 14, p. 202-223.

-, 2004, Reading sex in the eighteenth century: bodies and gender in English erotic culture, Cambridge, Cambridge University Press.

Hill Bridget, 1989, Women, work and sexual politics in eighteenth-century England, Oxford, B. Blackwell.

HiтcHсоск Tim, 1996, "Redefining sex in eighteenth-century England», History Workshop Journal, 41, p. 72-90.

—, 1997, English sexualities, 1700-1800, Basingstoke, Mac Millan.

—, 1999, "Sociability and misogyny in the life of John Cannon, 1684-1743 », in Michèle COHEN \& Tim HichCock (eds), 1999, English masculinities, 1660-1800, Londres, Longman, p. 25-43.

HunT Lynn (ed.), 1993, The invention of pornography: obscenity and the origins of modernity, 1500-1800, New York, Zone Books.

JACOB Margaret C., 1993, "The materialist world of pornography », in Lynn HunT (ed.), The invention of pornography: obscenity and the origins of modernity, 1500-1800, New York, Zone books. 
Jenner Mark S.R. \& Bertrand O. TAITHE, 2000, "The historiographical body ", in Roger COOTER \& John PICKSTONE (eds), Medicine in the twentieth century, Amsterdam, Harwood Academic Publishers (rééd. Londres, Routledge, p. 187200).

JORDANOVA Ludmilla, 1989, Sexual visions: images of gender in science and medicine between the eighteenth and nineteenth centuries, Londres, Horvester Wheatsheaf.

—, 1999, Nature displayed: gender, science and medicine, 1760-1820, Londres, Longman.

KENT Susan Kingsley, 1996 "Mistrials and diatribulations: a reply to Joan Hoff », Women's History Review, 5, p. 9-18.

KRAAKMAN Dorelies, 1994, "Reading pornography anew: a critical history of sexual knowledge for girls in French erotic fiction, 1750-1840 », Journal of the History of Sexuality, vol. 4, 4, p. 517-548.

LAQUeur Thomas, 1986, "Orgasm, generation, and the politics of reproductive biology », Representations, 14, p. 1-41.

-, 1990, Making sex : body and gender from the Greeks to Freud, Cambridge. Traduction française: 1992, La Fabrique du sexe. Essai sur le corps et le genre en Occident, traduction de Michel Gautier, Paris, Gallimard.

LILIEQUIST Jonas, 1991, "Peasants against nature: crossing the boundaries between man and animal in seventeenth- and eighteenth-century Sweeden ", Journal of the History of Sexuality, vol. 1, 3, p. 393-423.

MARTENSEN Robert, 1994, "The transformation of Eve: women's bodies, medicine and culture in early modern England », in Roy PORTER and Mikulas TeICH, Sexual knowledge, sexual science, Cambridge, Cambridge University Press, p. 107-133.

MCKENDrick Neil, Brewer John \& J.H Plumb., 1982, The birth of a consumer society: the commercialisation of eighteenth-century England, Londres, Hutchinson.

MCLAREN Angus, 1985, "The pleasures of procreation: traditional and biomedical theories of conception », in W.F. BYNUM \& Roy PORTER (eds), William Hunter and the eighteenth-century medical world, Cambridge, Cambridge University Press, p. 323341.

MCNAY Lois, 1992, Foucault and feminism: power, gender and the self, Cambridge, Polity Press.

MERCHANT Carolyn, 1980, The death of nature: women, ecology, and the scientific revolution, San Francisco, Harper and Row.

Milligan Don, 1993, Sex-life: a critical commentary on the history of sexuality, Londres, Pluto Press.

Moscucci Ornella, 1996, "Clitoridectomy, circumcision, and the politics of sexual pleasure in mid-Victorian Britain », in Andrew H. Miller \& James Eli ADAMS (eds), Sexualities in Victorian Britain, Bloomington and Indianapolis, Indianapolis Press, p. 60-78.

NEAD Lynda, 1988, Myths of sexuality: representations of women in Victorian Britain, Oxford, B. Blackwell. 
NEWMAN Louise M., 1991, "Critical theory and the history of women: what's at stake in deconstructing women's history », Journal of Women's History, 2, p. 58-68.

Ogborn Miles, 1998, Spaces of modernity: London's geographies, 1680-1780, New York, Guilford Press.

PERRY Ruth, 1992, "Colonising the breast: sexuality and maternity in eighteenthcentury England », in John C. FOUT (ed.), Forbidden history: the state, society, and the regulation of sexuality in modern Europe, Chicago, University Chicago Press (réimpression du JHS, vol. 2, 2, 1991, p. 204-235).

Peterson M. Jeanne, 1986, «Dr Acton's enemy: medicine, sex and society in Victorian England », Victorian Studies, 29, p. 569-590.

PINCHBECK Ivy, 1930, Women workers and the industrial revolution, 1750-1850, Londres, Routledge (rééd. Londres, F. Cass, 1981).

PORTER Roy, 1982a, English society in the eighteenth century, Londres, Allen Lane.

—, 1982b, "Mixed feelings: the Enlightenment and sexuality in eighteenth-century Britain ", in Paul-Gabriel BouCÉ (ed.), Sexuality in eighteenth-century Britain, Manchester, Manchester University Press.

—, 1987, "'The secrets of generation display'd": Aristotle's Master-piece in eighteenth-century England", in Robert PURKS MACCUBBIN (ed.), 'Tis nature's fault: unauthorized sexuality during the Enlightenment, Cambridge, Cambridge University Press, p. 1-21.

PuRvis June, 1996, "Women's history and poststructuralism », Women's History Review, 5, p. 5-7.

RILEY Denise, 1988, "Am I that name?": feminism and the category of "women" in history, Londres, Mac Millan.

ROPER Lyndal, 1994, Oedipus and the Devil: witchcraft, sexuality and religion in early modern Europe, Londres, Routledge.

RussETT Cynthia Eagle, 1989, Sexual science: the Victorian construction of womanhood, Cambridge, MA, Harvard University Press.

SCHIEBINGER Londa, 1987, "Skeletons in the closet: the first illustrations of the female skeleton in eighteenth-century anatomy », in Catherine GALLAGHER \& Thomas LAQUEUR (eds), The making of the modern body: sexuality and society in the nineteenth century, Berkeley, University of California Press.

—, 1989, The mind has no sex? Women in the origins of modern science, Cambridge, MA, Harvard University Press.

—, 1993, Nature's body: gender in the making of modern science, Boston, Beacon Press.

—, 1996, "Gender and natural history », in Nicholas JARDINE, James A. SECORD and Emma C. SPARY (eds), Cultures of natural history, Cambridge, Cambridge University Press, p. 171-187.

SCOTT Joan Wallach, 1988 (rééd. 1999), Gender and the politics of history, New York, Columbia University Press.

SENELICK Laurence, 1990, "Mollies or men of mode? Sodomy and the eighteenthcentury London stage ", Journal of the History of Sexuality, 1, p. 33-67. 
SHOEMAKer Robert B., 1998, Gender in English society, 1650-1850: the emergence of separate spheres?, Londres, Longman.

SNELL Keith, 1981, « Agricultural seasonal unemployment, the standard of living, and women's work in the South and East, 1690-1860 », Economic History Review, serie 2, 34, p. 407-437.

STONE Lawrence, 1979 [édition revue et corrigée] (1 ${ }^{\text {re }}$ éd. 1977), The family, sex and marriage in England, 1500-1800, Londres, Weidenfeld and Nicolson.

THACKER Andrew, 1997, «Foucault and the writing of history », in Moya LLOYD \& Andrew THACKER (eds), The impact of Michel Foucault on the social sciences and the bumanities, Basingstoke, Mac Millan.

THADDEus Janice Farrar, 1994, «Mary Delany, model to the age », in Beth FowKes TOBIN, History, gender and eighteenth-century literature, Athens, GA, University of Georgia Press.

TONKIN Boyd, 1996 (13 décembre), «Bodice heat », The Independent : Tabloid, p. 2-3.

TosH John, 1999, «The old Adam and the new man: emerging themes in the history of English masculinities », in Michèle COHEN \& Tim HicHCOCK, 1999, English masculinities, 1660-1800, Londres, Longman.

TRumbaCH Randolph, 1989, "The birth of the queen: sodomy and the emergence of gender equality in modern culture, 1660-1750 », in Martin DubERMAN, Martha VICINUS \& George CHAUNCY Jr. (eds), Hidden from history: reclaiming the gay and lesbian past, New York, New York American Library, p. 129-140.

- 1991, "Sex, gender, and sexual identity in modern culture: male sodomy and female prostitution in Enlightenment London », Journal of the History of Sexuality, vol. 2, 2, p. 186-203.

- 1998, Sex and the gender revolution. 1. Heterosexuality and the third gender in Enlightenment London, Chicago, University Chicago Press.

VICKERY Amanda, 1993, "Golden age to separate spheres? A review of the categories and chronology of English women's history ", Historical Journal, 36, p. 383-414.

WAGNER Peter, 1988, Eros revived: erotica of the Enlightenment in England and France, Londres, Secker and Warburg.

WEEKS Jeffrey, 1989 (1 1 re éd. 1981), Sex, politics and society: regulation of sexuality since 1800, 2e éd., Londres, Longman. 